\section{IMI's leadoff hitter}

\section{By Kai-Jye Lou, Staff Writer}

Europe's Innovative Medicines Initiative has recruited 7 pharmas to each contribute up to 50,000 compounds from their proprietary libraries and is pooling the molecules into a public-private partnership called the European Lead Factory. The pharmas will have access to an expanded chemical space, and academic members of the factory will be able to run experiments with molecules that are much higher in quality than what is typically found in academia.

The five-year public-private partnership will have a $€ 169$ million (\$222.2 million) budget to help build a high throughput screening center and to assemble the library. IMI hopes to designate the site of the screening center by year end and to have it up and running by early 2013.

Under the partnership, participating member companies of the European Federation of Pharmaceutical Industries and Associations (EFPIA) will contribute up to 50,000 compounds each to populate the initial library with at least 300,000 molecules-called
"Ideally, what we really want to see 2-3 years into the partnership are projects that are identifying lead molecules that could be taken up and developed into actual drugs."

-Hugh Laverty,

Innovative Medicines Initiative internal pharma R\&D efforts.

"In addition, the generation of tool compounds for basic science research may cut back on selected properties needed for drug development, including, for example, aspects related to pharmacokinetics and patentability," Laverty told SciBX. "Thus, despite delivering valuable mechanistic insights into biological function, their direct impact on early pharma research efforts remains limited. The Lead Factory is an attempt to bridge this gap translating academia's cutting-edge science to quality hit compounds with pharmaceutical potential."

He said the European Lead Factory will address this deficiency in two ways. First, the partnership will provide its members with access to a library that should be far more comprehensive than those currently available to the individual parties.

"The public will get access to compounds in high-quality chemical libraries, and the pharmas themselves will get access to the chemical libraries of other pharmas as well as compounds generated through the partnership," said Laverty. "This partnership will help us move past the model where one company only screens its own library for compounds that could be useful in the few select disease areas that it works in."

Second, the partnership will provide its public members with a high throughput screening facility and the necessary resources to screen large compound libraries.

Given the proprietary nature of the compound libraries being provided by EFPIA members, all screening projects under the partnership will be carried out in a blinded fashion in which structural information on the Joint European Compound Collection.

EFPIA represents the pharmaceutical industry in Europe and was jointly launched by the IMI with the European Commission in 2008.

Once fully operational, the screening center is expected to support up to 24 high throughput screening projects from its non-EFPIA members, with up to 500,000 compounds per screen. The center also will provide its EFPIA members with full copies of the compound library for up to 24 additional screening projects. These projects will be run by the EFPIA members themselves.

The overarching goal of the European Lead Factory is to provide both pharma and academics with access to the tools and facilities needed to generate high-quality hits for drug R\&D.

"Ideally, what we really want to see $2-3$ years into the partnership are projects that are identifying lead molecules that could be taken up and developed into actual drugs," said Hugh Laverty, senior scientific project manager of IMI.

\section{Opening up pharma libraries}

Laverty said pharmas are becoming increasingly reliant on external sources such as research institutes, universities and smaller companies for early stage drug discovery and target research. The problem, he said, is that those sources often lack the resources, including suitable compound collections, to generate sufficient numbers of high-quality lead structures needed for such research. As a result, their productivity is low relative to library compounds is withheld. Only members of the screening center itself will have access to both bioactivity and chemical structural information for all the compounds in the Joint European Compound Collection.

For each screening project, including those carried out by EFPIA members themselves, the screening center will generate a qualified hit list of up to 50 compounds. Structural information for compounds in the qualified hit list will be unblinded and released only to the owners of the project.

The library copies that EFPIA members receive from the screening center will be blinded and will not include any structural data on the compounds. Thus, EFPIA members will need to submit the results from their in-house screening projects back to the center, which will then provide the member with a list of up to 50 qualified hits and the associated structural information.

Laverty said rights to existing IP, such as those that cover molecules in the compound library, will be retained by their respective owners.

Rights to new IP generated from projects carried out under the European Lead Factory partnership will be negotiated by the involved parties. Laverty said IMI has a guidance for IP-related issues in place.

\section{Call for proposals}

Under the European Lead Factory partnership, EFPIA members will 
collaborate on projects with public and private entities that are eligible for IMI funding, collectively referred to as the Applicant Consortia. This group will include universities, small- to medium-sized enterprises, patient organizations and regulatory agencies.

The partnership's EFPIA members are AstraZeneca plc, Bayer AG's Bayer HealthCare unit, Johnson \& Johnson's Janssen Pharmaceuticals Inc. unit, H. Lundbeck A/S, Merck KGaA's Merck-Serono S.A. unit, Sanofi and UCB Group.

Laverty said the call for proposals was just issued and the Applicant Consortia are in the process of being formed.

The call for proposals will occur in two stages in which members of the European Lead Factory's Applicant Consortia first submit an Expression of Interest that will be reviewed and ranked by an independent group of experts. Applicants with the highest-ranked proposals will receive an invitation to develop a full project proposal with the EFPIA Consortium, which will then be submitted for final review by an independent group. Final project proposals that receive a favorable review will receive IMI funding.

For members of the Applicant Consortia, up to $75 \%$ of the costs associated with research and technological development activities, and up to $100 \%$ of the direct costs associated with other activities such as management and training, will be eligible for IMI funding. There is a separate reimbursement schedule to cover indirect costs incurred by members of the Applicant Consortia, such as overhead. The EFPIA members themselves are not eligible for IMI funding and will be responsible for funding their own activities and projects carried out under the partnership.
Under the five-year partnership, the European Commission's 7th Framework Programme for Research will contribute up to $€ 80$ million ( $\$ 105.2$ million), with $€ 40$ million (\$52.6 million) each allocated for projects related to the screening center and compound library.

The remaining $€ 89$ million ( $\$ 117$ million) of the $€ 169$ million ( $\$ 222.2$ million) budget will primarily come in the form of in-kind contributions from EFPIA members. These contributions include the 300,000 compounds that the EFPIA members will donate to the Joint European Compound Collection, which are valued at $€ 60$ million (\$78.9 million) assuming an average cost of $€ 200$ (\$262.94) per donated compound. The scientific, technical and legal expertise EFPIA members provide to collaborators is valued at $€ 24$ million ( $\$ 31.6$ million). Direct cash contributions from EFPIA members are expected to be about $€ 5$ million (\$6.6 million).

Lou, K.-J. SciBX 5(13); doi:10.1038/scibx.2012.325

Published online March 29, 2012

\section{COMPANIES AND INSTITUTIONS MENTIONED}

AstraZeneca plc (LSE:AZN; NYSE:AZN), London, U.K.

Bayer AG (Xetra:BAY), Leverkusen, Germany

European Federation of Pharmaceutical Industries and Associations, Brussels, Belgium

Innovative Medicines Initiative, Brussels, Belgium

H. Lundbeck A/S (CSE:LUN), Copenhagen, Denmark

Johnson \& Johnson (NYSE:JNJ), New Brunswick, N.J.

Merck KGaA (Xetra:MRK), Darmstadt, Germany

Sanofi (Euronext:SAN; NYSE:SNY), Paris, France

UCB Group (Euronext:UCB), Brussels, Belgium 